\title{
Compressed Sensing Based Dynamic MR Image Reconstruction By Using 3D-Total Generalized Variation and Tensor Decomposition: k-t TGV-TD
}

Jucheng Zhang

Zhejiang University

Lulu Han

Zhejiang Sci-Tech University

Jianzhong Sun

Zhejiang University

Zhikang Wang

Zhejiang University

Wenlong Xu

China Jiliang University

Ling Xia

Zhejiang University

Mingfeng Jiang ( $\nabla$ m.jiang@zstu.edu.cn )

Zhejiang Sci-Tech University

\section{Research Article}

Keywords: dynamic Magnetic Resonance Imaging (dMRI), higher-order singular value decomposition (HOSVD), total generalized variation (TGV), sparse representation

Posted Date: November 15th, 2021

DOI: https://doi.org/10.21203/rs.3.rs-1060947/v1

License: (a) (1) This work is licensed under a Creative Commons Attribution 4.0 International License. Read Full License 


\title{
Compressed Sensing based Dynamic MR Image Reconstruction by Us- 1
} ing 3D-Total Generalized Variation and Tensor Decomposition: k-t 2

\section{TGV-TD}

Jucheng Zhang ${ }^{1^{\dagger}}$, Lulu Han ${ }^{2^{\dagger}}$, Jianzhong Sun ${ }^{3}$, Zhikang Wang ${ }^{1}$, Wenlong Xu ${ }^{4}$, Ling Xia ${ }^{5}$, Mingfeng Jiang ${ }^{2 *}$

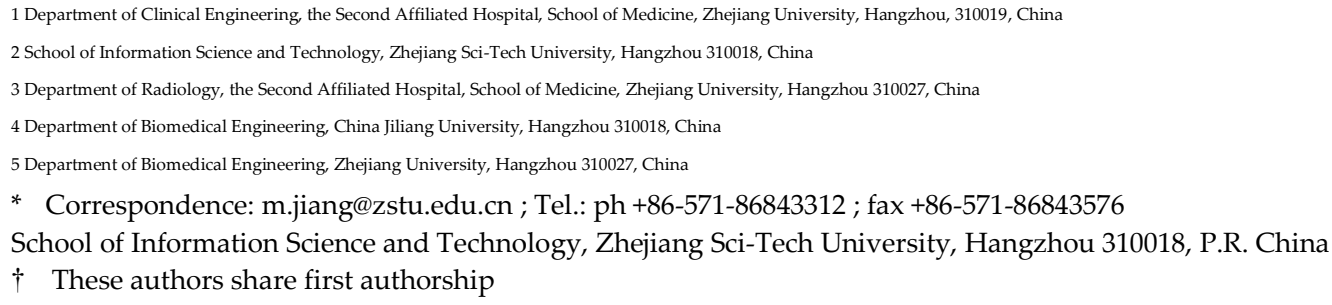

\begin{abstract}
:
Purpose: Compressed Sensing Magnetic Resonance Imaging (CS-MRI) is a promising technique to accelerate dynamic cardiac MR imaging (DCMRI). For DCMRI, the CS-MRI usually exploits image signal sparsity and low-rank property to reconstruct dynamic images from the undersampled k-space data. In this paper, a novel CS algorithm is investigated to improve dynamic cardiac MR image reconstruction quality under the condition of minimizing the k-space recording.

Methods: The sparse representation of 3D cardiac magnetic resonance data is implemented by synergistically integrating 3D TGV algorithm and high order singular value decomposition (HOSVD) based Tensor Decomposition, termed as k-t TGV-TD method. In the proposed method, the low rank structure of the 3D dynamic cardiac MR data is performed by the HOSVD method, and the localized image sparsity is achieved by the 3D TGV method. Moreover, the Fast Composite Splitting Algorithm (FCSA) method, combining the variable splitting with operator splitting techniques, is employed to solve the low-rank and sparse problem. Two different cardiac MR datasets (cardiac cine and cardiac perfusion MR data) are used to evaluate the performance of the proposed method.
\end{abstract}

Results: Compared with the state-of-art methods, such as the k-t SLR method, 3D TGV method and HOSVD based tensor decomposition method, the proposed method can offer improved reconstruction accuracy in terms of higher signal-to-error ratio (SER).

Conclusions: This work proved that the k-t TGV-TD method was an effective sparse representation way for DC-MRI, which was capable of significantly improving the reconstruction accuracy with different reduction factor.

Keywords: dynamic Magnetic Resonance Imaging (dMRI), higher-order singular value decomposition (HOSVD), total generalized variation (TGV), sparse representation.

\section{Introduction}

In Magnetic Resonance Imaging (MRI), imaging speed is limited by slow acquisition of full k-space using magnetic field gradients [1] . Minimizing the k-space recording time without compromising image quality has been a main thrust of MR imaging research. With the advent of compressed sensing (CS) theory [2,3], MR image reconstruction with 
sparsity-promoted regularization (e.g., $\ell_{1}$-based regularization), termed as CS-MRI[4-10], has gained popularity for its high imaging speed. The effective exploitation of the signal sparsity enables the MR image reconstruction from far fewer k-space samples possible than conventional methods require, thus CS-MRI can significantly reduce the scan time. The compressed sensing theory has been successfully applied to both static and dynamic magnetic resonance imaging (dMRI) reconstructions [11-14].

In CS-MRI, the method used to sparsify the MR image plays an important role in the image reconstruction. The most used sparsity bases are predefined mathematical transforms, such as discrete cosine transform (DCT), and discrete wavelet transform (DWT). Recently, the singular value decomposition (SVD) method has been used as a data-adaptive sparsity basis in CS-MRI reconstruction [15,16], and it has been found that the SVD-based method could significantly accelerate the reconstruction process and achieve better image quality than those commonly used sparsifying transforms (DCT and DWT). Majumdar et al. proposed to exploit the nuclear norm regularization to implement the CS-MRI reconstruction, and the results showed that the proposed reconstruction method was faster than other methods[6] . In addition, the linear combination of Total Variation (TV) and wavelet sparse regularization, known as TV-L1 problem, is very popular in many CS-MRI models[5,6,17], which can be considered as processing the MR image to be sparse by both the specific transform and finite-differences at the same time. Due to the stair-case artifacts caused by the conventional TV-based regularization[18,19], several generalizations and extensions of TV have been introduced to improve the CS-MRI reconstruction accuracy, such as Total Generalized Variation (TGV)[18-20] , Higher Degree Total Variation (HDTV) [21]. Nonlocal Total Variation (NLTV) [22-24] is another effective way to address the issue of stair-case artifacts. Although effective in practice, it involves higher computational complexity than the conventional TV method.

For dynamic MR image reconstruction, Ji, et al. adopted the difference between the reconstructed image and the reference image to represent the spatial sparsity [25]. However, when compared with the reference time frame, the sparsity of the difference image got worse with the increase of the subsequent frame distance. To solve this problem, Majumdar took the difference between two adjacent sub-images as a sparse representation of the reconstructed MR image [13]. In addition, Usman put forward the concept of a sparse group of dynamic MRI, utilizing both MRI signal itself sparsity and the group structure information between signals [26,27], which can effectively improve the image reconstruction quality. Moreover, a novel blind compressed sensing frame work was proposed to recover dynamic magnetic resonance images from undersampled measurements $[28,29]$, which has been proved to provide superior reconstruction performance in comparison to existing low rank and compressed sensing schemes. Recently, $k-t$ SLR ( $k-t$ Sparisity and Low-Rank) method has been proposed to accelerate dynamic MRI by exploiting sparsity and low rank properties of the image data[30,31] . To exploit the low-rank structure, the $k$ - $t$ SLR method reshaped the 3D dataset into a large 2D matrix through a two-step process: vectorize the $2 \mathrm{D}$ images in a dynamic sequence first and then concatenate them to form a matrix. In most of the existing dynamic CS-MRI methods, 2D/1D transforms were applied to solve the 3D dynamic problem, which, by treating the 3D data as a series of 2D images, unfolded the 3D dataset into a 2D matrix to explore the spatiotemporal redundancy [30-33]. In addition, Majumdar [34,35] acted the dynamic MR image reconstruction problem as a least squares minimization regularized by $l p$-norm as the sparsity penalty and Schatten- $q$ norm as the low-rank penalty sparsity, which can yield much better reconstruction results than k-t SLR method. However, reshaping a high-order tensor into a matrix or vector may neglect the inherent data redundancy, thus greatly degrading the reconstructed image quality. To promote the signal sparsity representation by exploring the redundancy of the high-dimension data format, $\mathrm{Yu}$ et al proposed tensor decomposition-based sparsifying transform, that is, high-order Singular Value Decomposition (HOSVD)[36], which can outperform the 
conventional sparse recovery methods for high-dimensional cardiac imaging reconstruction accuracy given the same amount of k-space data set[37] .

In this paper, we will further improve the HOSVD based CS-MRI method to synergistically integrate 3D TGV algorithm and HOSVD-based Tensor Decomposition, termed as k-t TGV-TD method. In the proposed method, the low rank structure of the 3D dynamic cardiac MR data is performed by the HOSVD method, and the localized image sparsity is achieved by the 3d-TGV method. Meanwhile, the Fast Composite Splitting Algorithm (FCSA) method[6], combining the variable splitting with operator splitting techniques, is employed to solve the low-rank and sparse problem [38]. Two different cardiac MR datasets (cardiac cine and cardiac perfusion MR data) are used to evaluate the performance of the proposed method.

\section{Theory of k-t TGV-TD Method}

In the proposed CS-MRI technique, 3d-TGV and HOSVD based tensor decomposition are used to promote the sparsity of the dynamic MR signals, and the k-t TGV-TD optimization problem can be formed as:

$$
\underset{\chi}{\arg \min }\left\{\left\|A_{u}(\chi)-b\right\|_{2}^{2}+\lambda_{1} \cdot T G V_{\alpha}^{2}(\chi)+\lambda_{2} \varphi(\chi)\right\}
$$

where $\boldsymbol{A}_{\boldsymbol{u}}$ is undersampled Fourier operator of the MR image, $\boldsymbol{b}$ is undersampled measurement of $k$-space data, and $\chi$ is a third order tensor used to represent the spatial-temporal 3D cardiac MR data. $\lambda_{1}$ and $\lambda_{2}$ are two positive regularization parameters that determine the trade-off between the data consistency and the sparsity regularization terms. $\varphi(\chi)$ is the tensor decomposition, and $T G V_{\alpha}^{2}(\chi)$ is the second order TGV penalty function. A detailed introduction about 3d-TGV method and HOSVD based tensor decomposition method can be found in the Appendix A and B respectively.

Fast composite splitting algorithm processes the original and composite regularization problem into two simpler sub-problems, which are then solved by using the fast iterative shrinkage-threshold algorithm (FISTA). In this way, we could finally reconstruct the dynamic images via an iterative combination [39]. Specifically, the complex composite reconstruction problem in Eq. (1) can be decomposed into two simpler regularization subproblems, that is, TGV subproblem and TD subproblem, as shown in Eqs. (2) and (3):

$$
\begin{aligned}
& \underset{\chi}{\arg \min }\left\{\frac{1}{2}\left\|A_{u}(\chi)-b\right\|_{2}^{2}+\lambda_{1} \cdot T G V_{\alpha}^{2}(\chi)\right\} \\
& \underset{\chi}{\arg \min }\left\{\frac{1}{2}\left\|A_{u}(\chi)-b\right\|_{2}^{2}+\lambda_{2} \varphi(\chi)\right\}
\end{aligned}
$$

The basic idea of FISTA is to build regularization for the linearized differentiable part of the objective function in each iteration[38-40] . So, the subproblem Eqs. (2) and (3) can be extended into two parts respectively:

$$
\min \left\{F(\chi) \equiv f(\chi)+g(\chi): \chi \in C^{I_{1} \times I_{2} \times I_{3}}\right\}
$$


where $f(\chi)=\frac{1}{2}\left\|A_{u}(\chi)-b\right\|_{2}^{2}$ is a smooth convex function which is continuously dif132 ferentiable with Lipschitz constant $L_{f}$ (usually large); and $g(\chi)=\lambda_{1} \cdot T G V_{\alpha}^{2}(\chi)$ or 133 $\lambda_{2} \varphi(\chi)$ is a continuous convex function which is nonsmooth. According to the FISTA algorithm, given a continuous function $g(u)$ and any scalar $L>0$, the proximal map associated with function $g(\chi)$ can be built as follows:

$$
\operatorname{prox}_{L}\{g(u), \chi\}=\underset{u}{\arg \min }\left\{g(u)+\frac{L}{2}\|u-\chi\|^{2}\right\}
$$

Eq. (2) and Eq. (3) are solved in an iterative fashion. Let $X_{1}$ be the solution of the TGV subproblem Eq. (2) and $X_{2}$ be the solution of the TD subproblem Eq. (3) respectively; in each $k$ iteration, the solutions $\chi_{k}$ to the overall problem Eq. (1) can be found by a linear combination as follows:

$$
\chi_{k}=\frac{1}{2}\left(X_{1}+X_{2}\right)
$$

The FCSA-based algorithm for solving the $k-t$ TGV-TD problem-based CS- $d$ MRI reconstruction can be described in the algorithm 1.

Algorithm 1. FCSA-based algorithm for solving the $k-t$ TGV-TD problem-based CS- $d$ MRI reconstruction

$$
\begin{aligned}
& \text { Initialize } L=L_{f}, \lambda_{1}, \lambda_{2}, r_{1}=\chi_{0}, t_{1}=1 \\
& \begin{array}{l}
\text { while }\left\|\chi_{n}-\chi_{n-1}\right\|_{2} /\left\|\chi_{n}\right\|_{2}>\text { tol or } k<\text { maxiter } d o \\
u=r_{k}-\frac{1}{L} \nabla f\left(r_{k}\right) \\
X_{1}=\operatorname{prox}_{L}\left\{\lambda_{1} \cdot T G V_{\alpha}^{2}(u), \chi\right\} \\
X_{2}=\operatorname{prox}_{L}\left\{\lambda_{2} \varphi(u), \chi\right\} \\
\chi_{k}=1 / 2\left(X_{1}+X_{2}\right) \\
t_{k+1}=\frac{1+\sqrt{1+4\left(t_{k}\right)^{2}}}{2} \\
r_{k+1}=\chi_{k}+\left(\frac{t_{k}-1}{t_{k+1}}\right)\left(\chi_{k}-\chi_{k-1}\right) \\
k \leftarrow k+1 \\
\text { end while }
\end{array}
\end{aligned}
$$

\section{Materials and Methods}

To test the reconstruction performance of the proposed k-t TGV-TD method, two different MR raw datasets, i.e. cardiac perfusion and cardiac cine, were employed in this study. The first dataset, a cardiac perfusion MR datasets, which was obtained on a 3T Siemens scanner with saturation recovery sequence $(\mathrm{TR} / \mathrm{TE}=2.5 / 1 \mathrm{~ms}$, saturation recovery 
time $=100 \mathrm{~ms}$ ) at the University of Utah, was used in the k-t SLR method[30] ${ }^{1}$. It contained 70 time series data, and the data from a single slice was acquired on a Cartesian grid with a k-space matrix of $90 \times 190$ (phase encoding $\times$ frequency encoding) at a temporal resolution of one heartbeat. The second dataset, in vivo cardiac cine MR data, which was acquired on a $1.5 \mathrm{~T}$ Philips system at Yonsei University Medical Center, was used in the k-t FOCUSS method [41,42] $]^{2}$. The datasets is composed of 25 frames of full k-space data. The matrix size for scanning is $256 \times 256$, which corresponds to 256 phase encoding steps and 256 samples in frequency encoding. The cine cardiac data was obtained using steady-state free precession (SSFP) sequence with a flip angle of 50 degree and TR=3.45 $\mathrm{ms}$. The FOV (Field of View) was $345 \mathrm{~mm} \times 270 \mathrm{~mm}$, and the slice thickness was $10 \mathrm{~mm}$. A representative frame and $x$ - $t$ plots of both the cardiac perfusion data and cardiac cine data are shown in Figure 1 and Figure 2 respectively. In addition, the radial sampling pattern was used to undersample the k-space of two datasets, which was simulated by rounding the sample locations to the nearest Cartesian location [30,41].

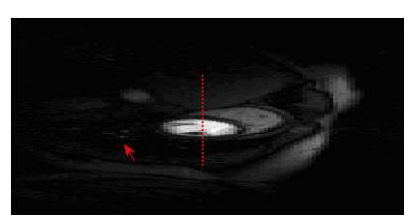

(a)

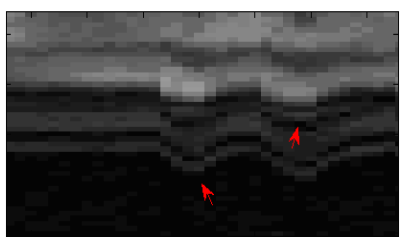

(b)

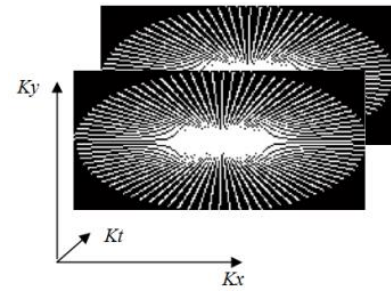

(c)

154

155

156

157

158

159

160

161

162

163

164

165

166

167

168

169

170

171

172 (a); (c) the radial under-sampling pattern with reduction factor 3.

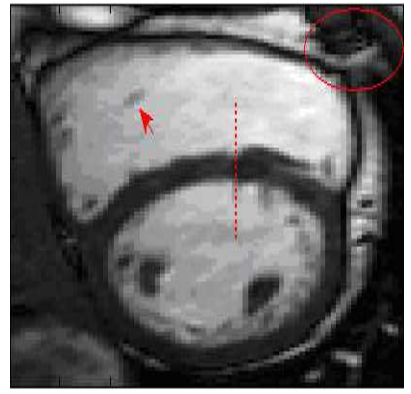

(a)

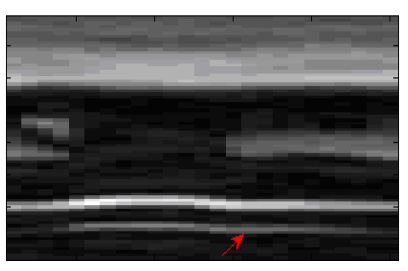

(b)

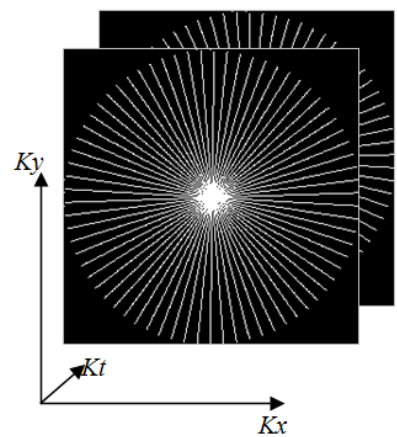

(c)

\section{3}

174

175 176 177

1 The cardiac perfusion data was acquired at the University of Utah and publicly available via web-link:

http://www.engineering.uiowa.edu/ jcb/Software/ktslr_matlab/Software.html

2 The MR data were acquired at Yonsei University Medical center in Korea and are publicly available via the web-link: https://bispl.weebly.com/k-t-focuss.html 
In each experiment, the regularization parameters $\lambda_{1}$ and $\lambda_{2}$ in the problem (1) were determined by parameter sweeping. The following stopping criteria were adopted for all experimental settings: the tolerance as shown in the FCSA-based reconstruction algorithm was set as $\boldsymbol{t o l}=10^{-4}$, and the maximum number of iterations was 30 . All reconstructions were implemented in the Matlab programming environment (Version 2009b, Mathworks, Natick, MA), and the experiments were performed on a ThinkPad laptop with $2.40 \mathrm{GHz}$ Intel Core 2 Duo processor, 4G of memory and Windows 7 operating system. In addition, we compared the proposed $k-t$ TGV-TD reconstruction method with three state-of-the-art dynamic CS-MRI reconstruction methods, that is, $k$ - $t$ SLR[30] , 3d-TGV method and HOSVD-based tensor decomposition method[37,43].

Moreover, the Signal-to-Error Ratio (SER) and computation cost were employed to quantitatively evaluate the accuracy and efficiency of the proposed dynamic CS-MRI reconstruction method. Furthermore, the reconstructed images, their error images (the absolute difference between reconstructed image and the full sampled MR image), and temporal profile $x$ - $t$ were also compared visually. The SER was formulated as follows $[30,37]$ :

$$
S E R=-10 \log _{10} \frac{\left\|m_{\text {recon }}-m_{\text {full }}\right\|_{F}^{2}}{\left\|m_{\text {full }}\right\|_{F}^{2}}
$$

Where $m_{\text {recon }}$ and $m_{\text {full }}$ denote the reconstructed and fully sampled gold standard images respectively, and $\|\mathrm{g}\|_{F}^{2}$ calculates the Frobenius norm.

\section{Results}

\section{A. Comparisons on the cardiac perfusion dataset}

The proposed $k$ - $t$ TGV-TD method was employed to reconstruct the cardiac perfusion data with variously reduced k-space sampling data. Figure 3 displays the performance evaluation of $k-t$ TGV-TD method in comparison with $\mathrm{k}-\mathrm{t}$ SLR, 3d-TGV and HOSVD methods on the cardiac perfusion MR dataset when reduction factor was 3 . The reconstructed MR image of one representative frame (19th frame), the $x$ - $t$ plots of the perfusion data (the spatial locations were indicated using dashed lines in the reconstructed MR image) and the error map were provided as a comparison. As can be seen in Figure 3, the proposed $k$ - $t$ TGV-TD method provided somewhat improvement in reducing artifacts, which was presented clearly in the error maps (the third column). The $x$ - $t$ plot of the reconstructions were illustrated in the second column of Figure 3 with reduction factor $R=3$. Each plots displayed the one-dimensional spatial profile, indicated by the dotted line in the first column, at all the temporal frames. It can be found that the reconstructions of $k-t$ SLR and HOSVD methods were smoothed, while the proposed method can capture the details of cardiac dynamics, indicating greater accuracy in temporal reconstruction.

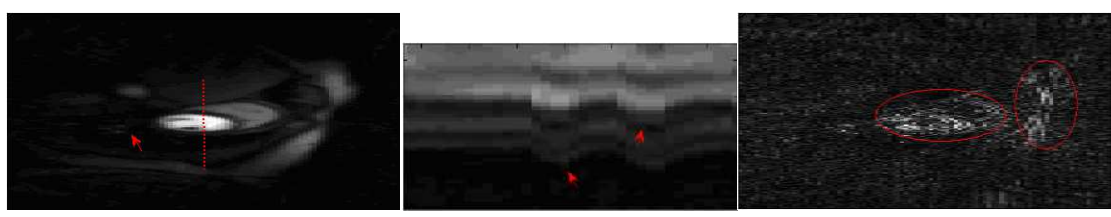

(a): $k-t$ SLR 


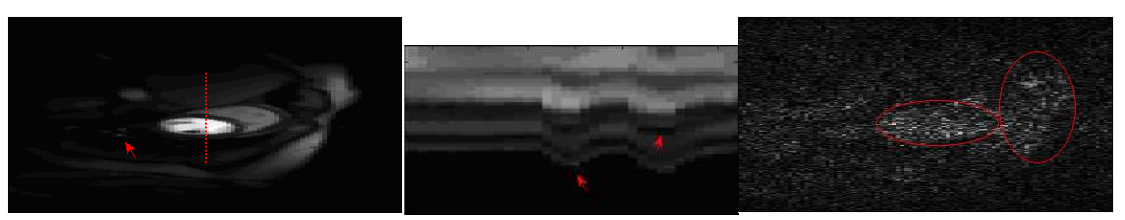

(b): 3-d TGV

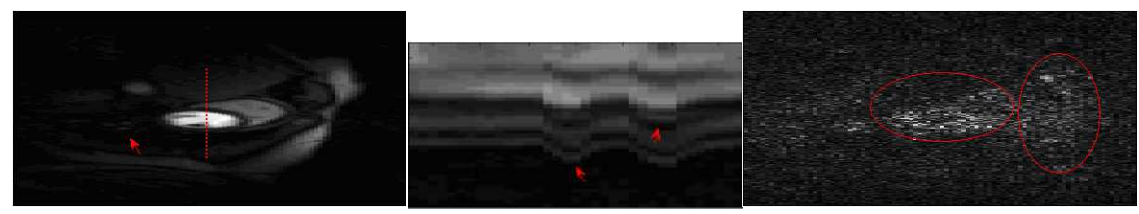

(c): HOSVD
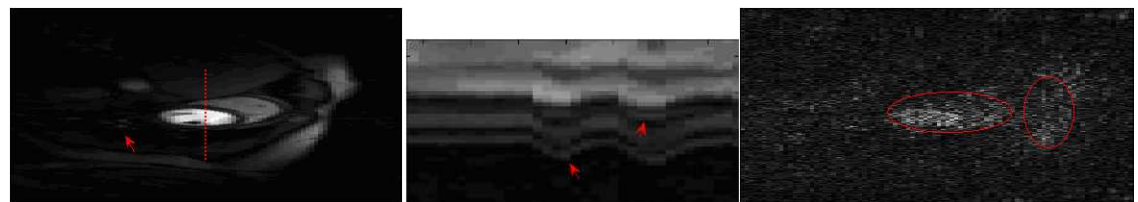

(d): k-t TGV-TD

Figure 3. Performance evaluation of $k-t$ TGV-TD method in comparison with $k-t$ SLR, 3-d TGV and HOSVD methods on the cardiac perfusion MR dataset. The first column is the reconstructed MR image with reduction factor 3; the second column is the $x$ - $t$ plots of the perfusion data, and the spatial locations were indicated using dashed lines in the first column; the third column is the error map. (a) $k$ - $t$ SLR method; (b)3-d TGV method; (c) HOSVD method; (d) $k-t$ TGV-TD method.

The SER of the reconstructed perfusion data are presented in Figure 4. In Figure 4, the proposed k-t TGV-TD method consistently provided higher SER than $k-t$ SLR, 3d TGV and HOSVD methods at all reduction factors $(2.5,3$ and 3.5 respectively, as shown in Figure 4(a-c)). Moreover, the 3d-TGV method slightly outperformed the HOSVD and $k-t$ SLR methods with better SER of each frame. In terms of the whole reconstructed image, the proposed method outperformed the $3 \mathrm{~d}-\mathrm{TGV}$ method by about $0.7 \mathrm{~dB}$, the HOSVD method by about $1 \mathrm{~dB}$, and the $k-t$ SLR method by about $1.5 \mathrm{~dB}$ respectively, as shown in Figure 4(d).

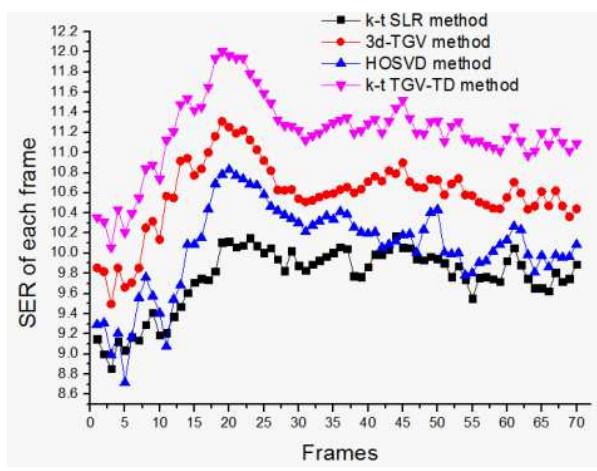

(a): $\mathrm{R}=2.5$

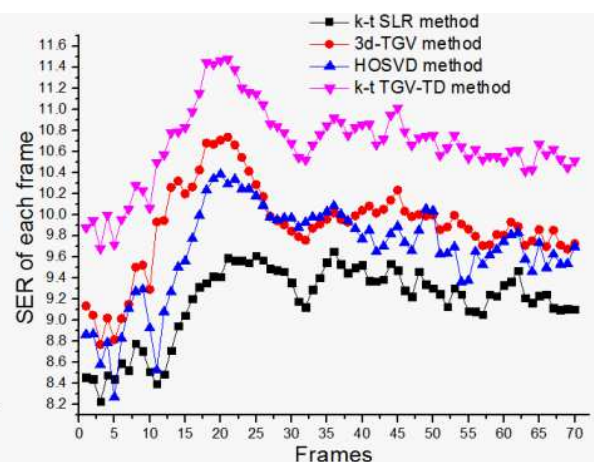

(b): $R=3$ 


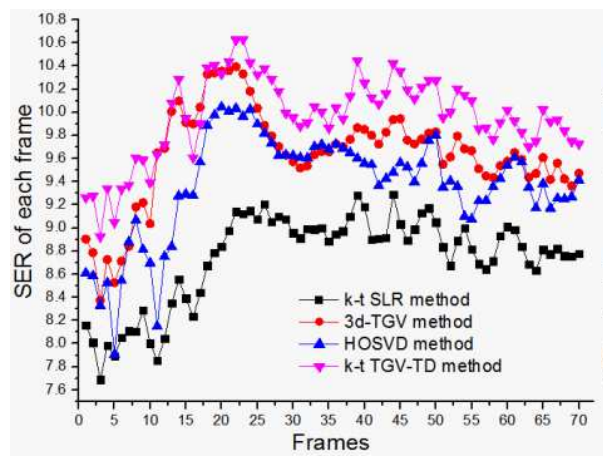

(c): $R=3.5$

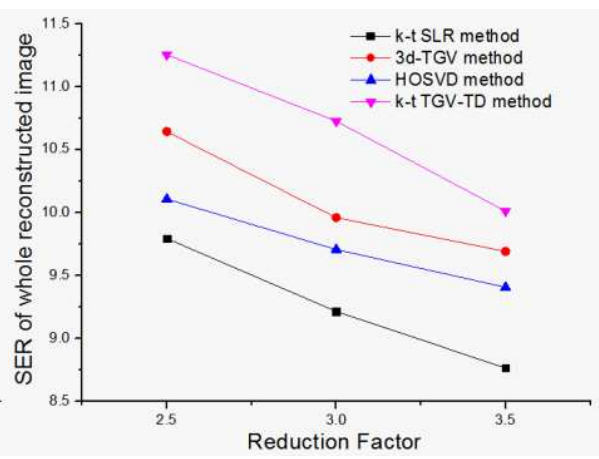

(d) place indexed by the red arrow in Figure 5(b).

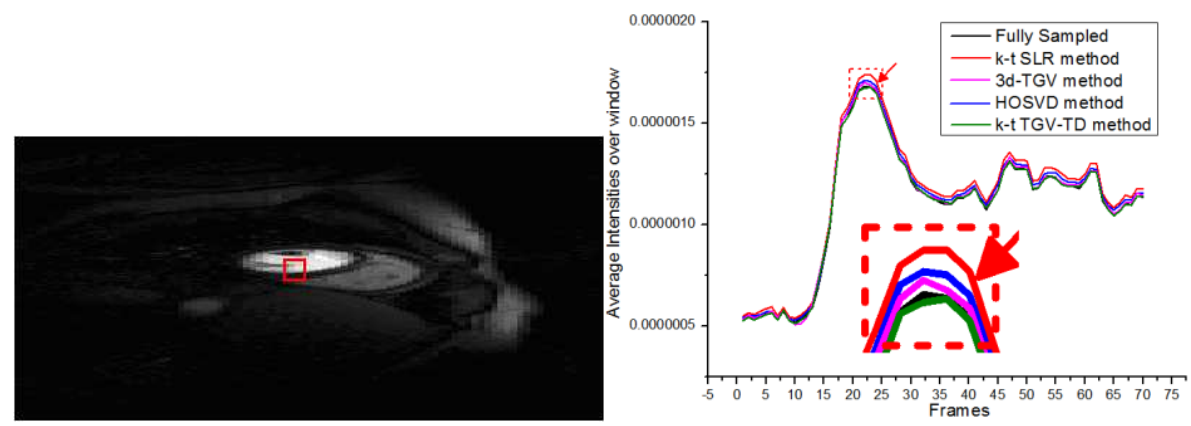

(a)

(b) error map. Visually, as shown in Figure 6, one can find that the proposed method outperformed the k-t SLR, 3d-TGV and HOSVD methods in reconstructing MR image with a 
little better defined borders (as shown in the red arrow in the first column) and less reconstruction artifacts (as shown in the third column). As can be seen in the second column of Figure 6, the $x$-t plots of the reconstructions by using the k-t SLR and HOSVD methods were contaminated by aliasing artifacts and noises, and the $x$-t plots of the reconstructions by using 3d-TGV method were over-smoothed.
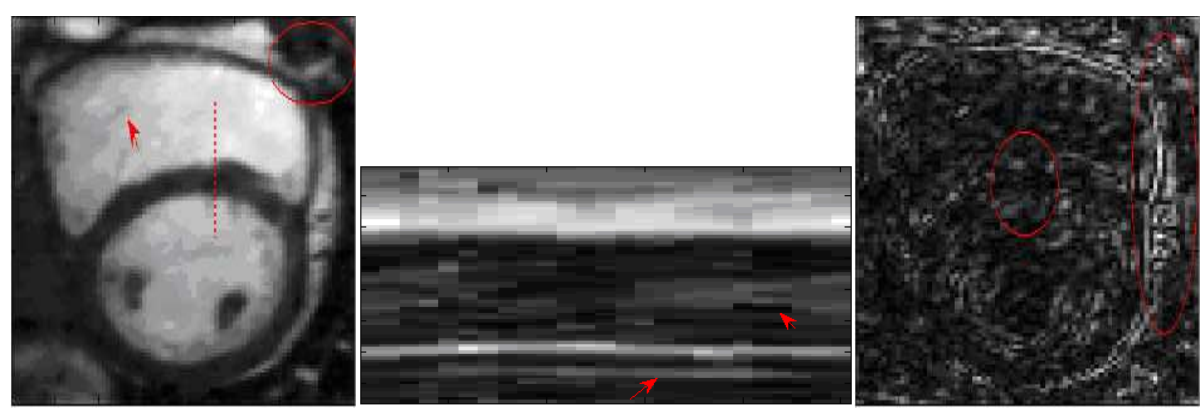

(a): k-t SLR method
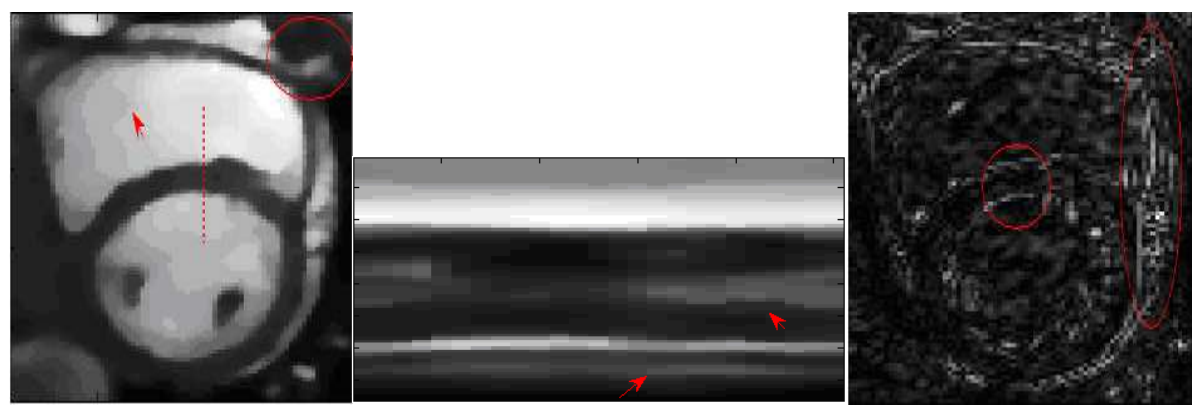

(b): 3D-TGV method
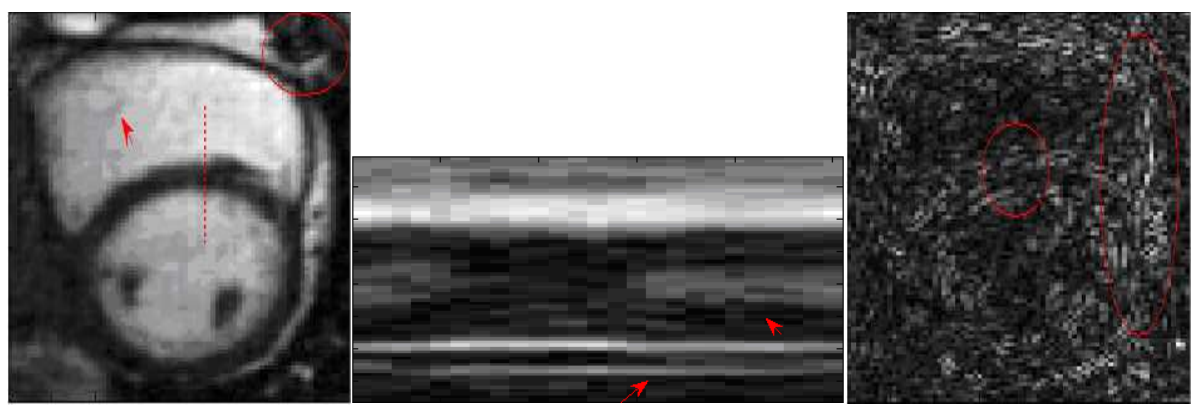

(c): HOSVD method
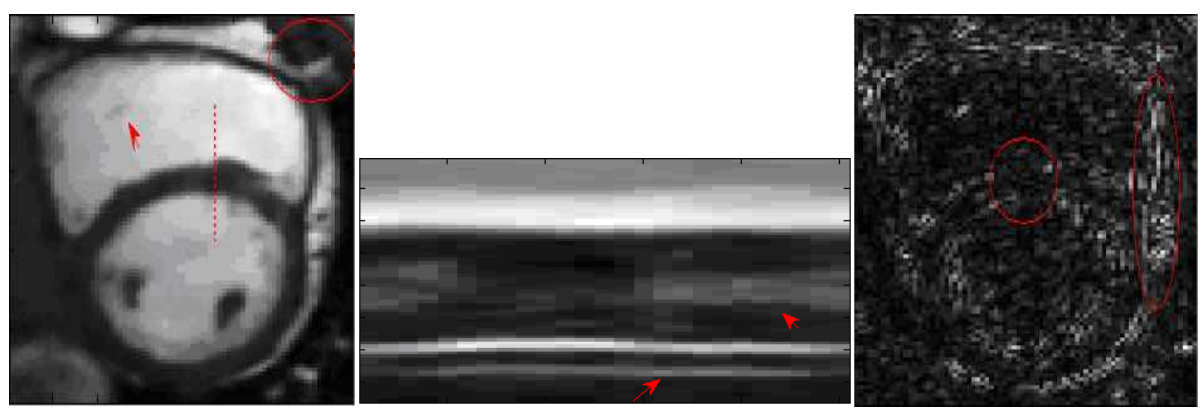

(d): k-t TGV-TD method

Figure 6. Performance evaluation of $k$ - $t$ TGV-TD method in comparison with $k$ - $t$ SLR, 3-d TGV and HOSVD methods on the cardiac cine MR dataset. The first column is the re- 
constructed MR image with reduction factor 8; the second column is the $x$ - $t$ plots of the cardiac cine data, and the spatial locations were indicated using dashed lines in the first column; the third column is the error map. (a) $k$ - $t$ SLR method; (b)3-d TGV method; (c) HOSVD method; (d) $k$ - $t$ TGV-TD method.

The performance comparisons between the proposed $k-t$ TGV-TD method and other reconstruction algorithms, i.e. $k$ - $t$ SLR method, 3d-TGV method and HOSVD method in terms of the SER frame by frame were presented in Figure 7. It can be found that the proposed method had the best reconstruction performances among all methods at all reduction factors (6, 8 and 10 respectively, as shown in Figure 7(a-c)). As shown in Figure 7(d), the proposed method outperforms the other methods to reconstruct the under-sampled cardiac cine MR image with higher SER on a voxel-by-voxel basis over entire frame at different reduction factors.

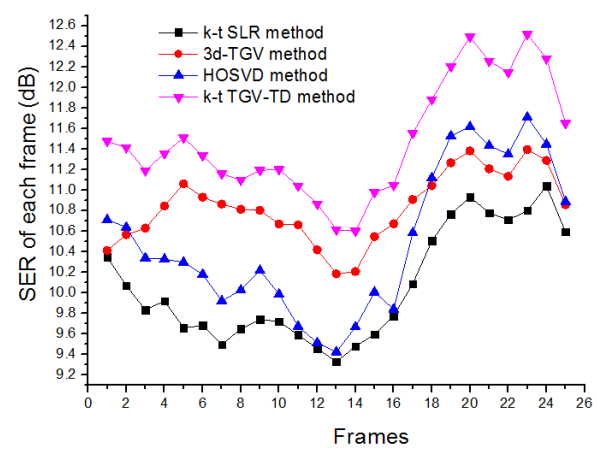

(a): $R=6$

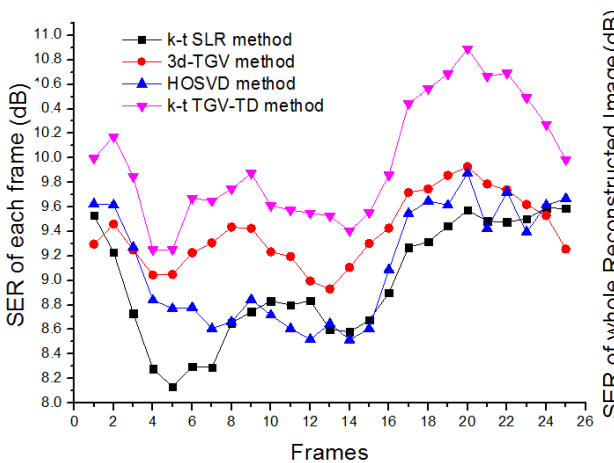

(c): $\mathrm{R}=10$

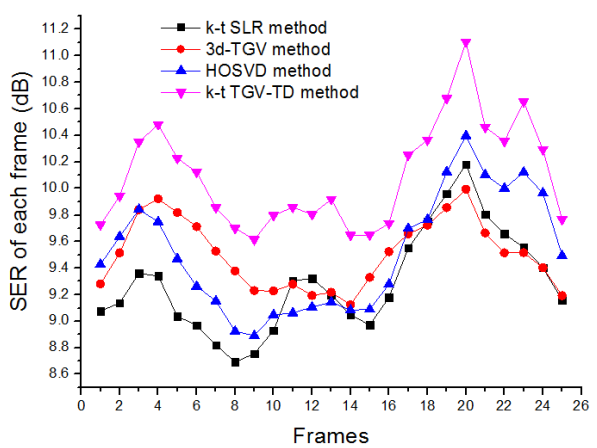

(b): $R=8$

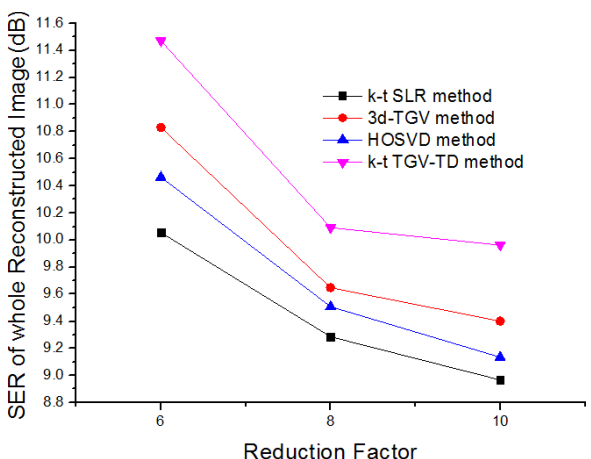

(d) 299 300 301 302 frame with different reduction factors.

As shown in Figure 8, the window located near posterior papillary muscle (red square in Figure 8(a)) was used to test the effects of artifacts on the temporal accuracy. It can be found that all the methods provided comparable averaged signal intensities for the posterior papillary muscle. However, when comparing the fully sampled myocardial signal intensities, the proposed $k-t$ TGV-TD method outperformed k-t SLR, 3d-TGV and HOSVD methods in capturing the cardiac signal intensity variation, especially in those frames indicated by the red arrows. Moreover, the large deviations between the fully sample gold standard and the reconstructions of k-t SLR, 3d-TGV and HOSVD methods can be observed easily, as shown in Figure 8 (b).
296

297

298 


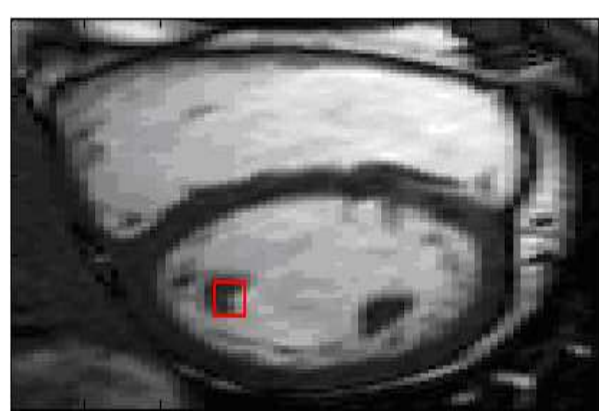

(a)

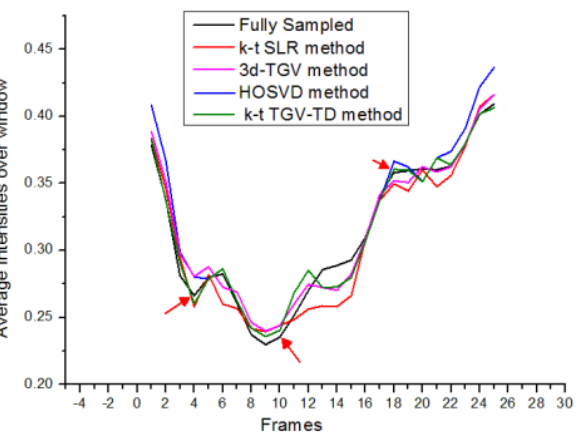

(b) accuracy.

From the reconstruction results, as shown in Figures 3 and 6, it can be observed that the proposed method can outperform the $k-t$ SLR, 3d-TGV and HOSVD methods in the investigated different dynamic cardiac MR image reconstructions. The $k$ - $t$ TGV-TD method can reconstruct the MR images with less error artifacts than those by using $k-t$ SLR and HOSVD methods. From the reconstruction accuracy SER and the average signal intensities of the specified window, it can be seen that advantages of the proposed method over its counterpart were apparent. The proposed method can get higher SER in each and entire frames at different reduction factors, and can reconstruct the pixel intensities averaged over the specified window with less deviation from the fully sampled gold standard. However, the computation cost of the proposed method is much more expensive than the k-t SLR and HOSVD methods, and a little bit higher than the 3d-TGV method, as shown in Figure 9. The longer reconstruction time of the proposed method can be attributed to exploiting the integration of HOSVD and 3D TGV sparsity constraints. In the future work, the overall reconstruction time of the proposed method can be reduced by the graphics processing unit (GPU) computing, which is expected to yield 30-50 times speed-up[43]. 


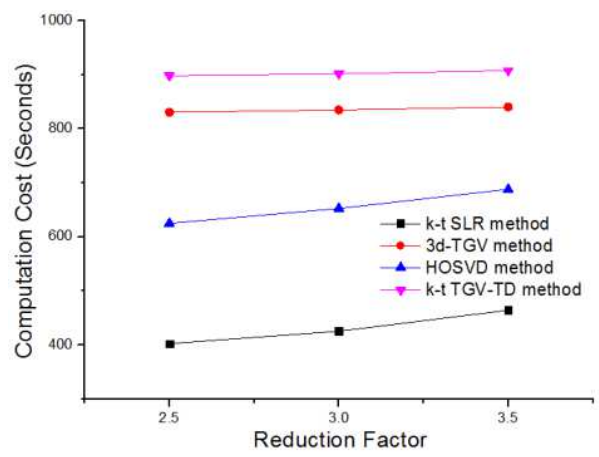

(a)

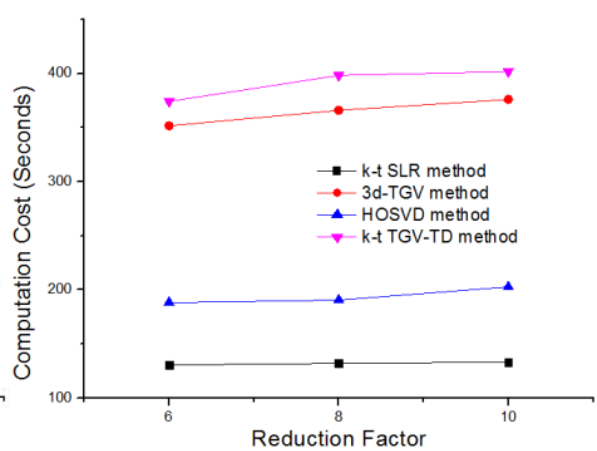

(b)
Figure 9. The computation cost of different algorithms with
(a) for cardiac perfusion dataset; (b) for cardiac cine dataset.

In this work, the proposed $k-t$ TGV-TD method explored the correlations and sparsity of the dynamic cardiac datasets, but did not integrate with partially parallel imaging (PPI). In addition, a combination of compressed sensing and parallel imaging was proposed to reconstruct the MR image[44,45], which can further reduce the k-space acquisition. In the future work, we will consider combining the $k$ - $t$ TGV-TD method with the PPI reconstruction method to further improve dynamic cardiac MR image reconstruction quality at higher reduction factors.

\section{Conclusion}

In this paper, a novel technique, called k-t TGV-TD, that combines the tensor decomposition and 3D total generalized variation (TGV), was proposed for dynamic MR imaging. The method was tested with cardiac perfusion and cardiac cine MR image reconstructions. The experimental results indicated that, compared with the k-t SLR, 3d-TGV and the HOSVD methods, the proposed method could achieve improved reconstruction accuracy in all the cases under investigation.

\section{Abbreviations}

MRI: Magnetic Resonance Imaging

DCMRI: dynamic cardiac MR imaging

CS: Compressed Sensing

dMRI: dynamic magnetic resonance imaging

HOSVD: high order singular value decomposition

SER: signal-to-error ratio

TV: Total Variation

TGV: total generalized variation

DCT: discrete cosine transform

DWT: discrete wavelet transform

SVD: singular value decomposition

49

50

351


HDTV: Higher Degree Total Variation

NLTV: Nonlocal Total Variation

k-t SLR: k-t Sparisity and Low-Rank method

FCSA: Fast Composite Splitting Algorithm

SSFP: steady-state free precession

FOV: Field of View

FISTA: fast iterative shrinkage-threshold algorithm

Ethics approval and consent to participate

Not applicable.

Consent for publication

Not applicable.

Availability of data and materials

The cardiac perfusion data was acquired at the University of Utah and publicly available

via web-link:

http://www.engineering.uiowa.edu/ jcb/Software/ktslr_matlab/Software.html

The MR data were acquired at Yonsei University Medical center in Korea and are

\section{Competing interests}

The authors declare that they have no competing interests.

\section{Funding}

This work is supported in part by the National Natural Science Foundation of China (61672466, 62011530130), in part by Joint Fund of Zhejiang Provincial Natural Science Foundation (LSZ19F010001), in part by the Key Research and Development Program of Zhejiang Province (2020C03060, 2020C03016), and Science Technology Department of Zhejiang Province (LGC19H180001).

\section{Author information}

\section{Authors' contributions}

JZ \& LH designed the k-t TGV-TD reconstruction method. JS, ZW, WX, LX and MJ contributed to the algorithm development and data analysis. All authors have been involved in drafting and revising the manuscript and approved the final version to be published. All authors read and approved the final manuscript. 
Correspondence to Mingfeng Jiang. m.jiang@zstu.edu.cn ; Tel.: ph +86-571-86843312 ; fax $+86-571-86843576$

Acknowledgements

The authors would like to thank S.G. Lingala for sharing the k-t SLR matlab code, and thanks Yu Y for providing the HOSVD sparsity basis code.

\section{References}

1. C. Westbrook, C.K.R.a.T.J. MRI in Practice (3rd edn). New York: Wiley-Blackwell 2006.

2. Donoho, D.L. Compressed sensing. IEEE Transactions on Information Theory 2006, 52, 1289-1306, doi:10.1109/tit.2006.871582.

3. Lustig, M.; Donoho, D.L.; Santos, J.M.; Pauly, J.M. Compressed sensing MRI. IEEE signal processing magazine 2008, 25, 72-82.

4. Lustig, M.; Donoho, D.; Pauly, J.M. Sparse MRI: The application of compressed sensing for rapid MR imaging. Magn Reson Med 2007, 58, 1182-1195, doi:10.1002/mrm.21391.

5. Shahdloo, M.; Ilicak, E.; Tofighi, M.; Saritas, E.U.; Cetin, A.E.; Cukur, T. Projection onto Epigraph Sets for Rapid Self-Tuning Compressed Sensing MRI. IEEE Trans Med Imaging 2019, 38, 1677-1689, doi:10.1109/tmi.2018.2885599.

6. Yang, G.; Yu, S.; Dong, H.; Slabaugh, G.; Dragotti, P.L.; Ye, X.; Liu, F.; Arridge, S.; Keegan, J.; Guo, Y. DAGAN: Deep de-aliasing generative adversarial networks for fast compressed sensing MRI reconstruction. IEEE transactions on medical imaging 2017, 37, 1310-1321.

7. Baron, C.A.; Dwork, N.; Pauly, J.M.; Nishimura, D.G. Rapid compressed sensing reconstruction of 3D non-Cartesian MRI. Magnetic resonance in medicine 2018, 79, 2685-2692.

8. Ye, J.C. Compressed sensing MRI: a review from signal processing perspective. BMC Biomedical Engineering 2019, 1, 1-17.

9. Bustin, A.; Fuin, N.; Botnar, R.M.; Prieto, C. From compressed-sensing to artificial intelligence-based cardiac MRI reconstruction. Frontiers in cardiovascular medicine 2020, 7, 17.

10. Delattre, B.M.A.; Boudabbous, S.; Hansen, C.; Neroladaki, A.; Hachulla, A.-L.; Vargas, M.I. Compressed sensing MRI of different organs: ready for clinical daily practice? European radiology 2020, 30, 308-319.

11. Liu, Y.; Liu, Q.; Zhang, M.; Yang, Q.; Wang, S.; Liang, D. IFR-Net: Iterative feature refinement network for compressed sensing mri. IEEE Transactions on Computational Imaging 2019, 6, 434-446.

12. Sun, L.; Fan, Z.; Fu, X.; Huang, Y.; Ding, X.; Paisley, J. A deep information sharing network for multi-contrast compressed sensing MRI reconstruction. IEEE Transactions on Image Processing 2019, 28, 6141-6153.

13. Liu, Y.; Liu, T.; Liu, J.; Zhu, C. Smooth robust tensor principal component analysis for compressed sensing of dynamic MRI. Pattern Recognition 2020, 102, 107252.

14. Zhao, B.; Haldar, J.P.; Christodoulou, A.G.; Liang, Z.-P. Image reconstruction from highly undersampled (k,t)-space data with joint partial separability and sparsity constraints. IEEE Transactions on Medical Imaging 2012, 31, 1809-1820, doi:10.1109/tmi.2012.2203921.

15. Giese, D.; Schaeffter, T.; Kozerke, S. Highly undersampled phase-contrast flow measurements using compartment-based k-t principal component analysis. Magn Reson Med 2013, 69, 434-443, doi:10.1002/mrm.24273.

16. Velikina, J.V.; Samsonov, A.A. Reconstruction of dynamic image series from undersampled MRI data using data-driven model consistency condition (MOCCO). Magn Reson Med 2015, 74, 1279-1290, doi:10.1002/mrm.25513.

17. Tsaig, Y.; Donoho, D.L. Extensions of compressed sensing. Signal Processing 2006, 86, 549-571, doi:10.1016/j.sigpro.2005.05.029.

18. Bredies, K.; Kunisch, K.; Pock, T. Total Generalized Variation. SIAM Journal on Imaging Sciences 2010, 3, 492-526, doi:10.1137/090769521. 
19. Knoll, F.; Bredies, K.; Pock, T.; Stollberger, R. Second order total generalized variation (TGV) for MRI. Magnetic Resonance in Medicine 2011, 65, 480-491, doi:10.1002/mrm.22595.

20. Guo, W.; Qin, J.; Yin, W. A New Detail-Preserving Regularization Scheme. SIAM Journal on Imaging Sciences 2014, 7, 1309-1334, doi:10.1137/120904263.

21. Hu, Y.; Jacob, M. Higher degree total variation (HDTV) regularization for image recovery. IEEE Trans Image Process 2012, 21, 2559-2571, doi:10.1109/TIP.2012.2183143.

22. Yang, J.H.F. Compressed magnetic resonance imaging based on wavelet sparsity and nonlocal total variation. IEEE International Symposium on Biomedical Imaging 2012.

23. Murphy, M.; Alley, M.; Demmel, J.; Keutzer, K.; Vasanawala, S.; Lustig, M. Fast $1_{1}$-SPIRiT compressed sensing parallel imaging MRI: scalable parallel implementation and clinically feasible runtime. IEEE Trans Med Imaging 2012, 31, 1250-1262, doi:10.1109/tmi.2012.2188039.

24. Qu, X.; Hou, Y.; Lam, F.; Guo, D.; Zhong, J.; Chen, Z. Magnetic resonance image reconstruction from undersampled measurements using a patch-based nonlocal operator. Med Image Anal 2014, 18, 843-856, doi:10.1016/j.media.2013.09.007.

25. Jim Ji, T.L. Dynamic MRI with compressed sensing imaging using temporal correlations. IEEE International Symposium on Biomedical Imaging 2008.

26. Usman, M.; Prieto, C.; Schaeffter, T.; Batchelor, P.G. k-t Group sparse: a method for accelerating dynamic MRI. Magn Reson Med 2011, 66, 1163-1176, doi:10.1002/mrm.22883.

Prieto, C.; Usman, M.; Wild, J.M.; Kozerke, S.; Batchelor, P.G.; Schaeffter, T. Group sparse reconstruction using intensity-based clustering. Magn Reson Med 2013, 69, 1169-1179, doi:10.1002/mrm.24333. S. G. Lingala, M.J. Blind compressive sensing dynamic MRI. IEEE Tran. Med. Imaging. 2013.

Majumdar, A. Improving synthesis and analysis prior blind compressed sensing with low-rank constraints for dynamic MRI reconstruction. Magn Reson Imaging 2015, 33, 174-179, doi:10.1016/j.mri.2014.08.031.

30. Lingala, S.G.; Hu, Y.; DiBella, E.; Jacob, M. Accelerated Dynamic MRI Exploiting Sparsity and Low-Rank Structure: k-t SLR. IEEE Transactions on Medical Imaging 2011, 30, 1042-1054, doi:10.1109/tmi.2010.2100850.

31. Lingala, S.G.; DiBella, E.; Adluru, G.; McGann, C.; Jacob, M. Accelerating free breathing myocardial perfusion MRI using multi coil radial k-t SLR. Phys Med Biol 2013, 58, 7309-7327, doi:10.1088/0031-9155/58/20/7309. Petrov, A.Y.; Herbst, M.; Andrew Stenger, V. Improving temporal resolution in fMRI using a 3D spiral acquisition and low rank plus sparse (L+S) reconstruction. Neuroimage 2017, 157, 660-674, doi:10.1016/j.neuroimage.2017.06.004.

33. Sun, A.; Zhao, B.; Li, Y.; He, Q.; Li, R.; Yuan, C. Real-time phase-contrast flow cardiovascular magnetic resonance with low-rank modeling and parallel imaging. J Cardiovasc Magn Reson 2017, 19, 19, doi:10.1186/s12968-017-0330-1.

34. Majumdar, A. Improved dynamic MRI reconstruction by exploiting sparsity and rank-deficiency. Magn Reson Imaging 2013, 31, 789-795, doi:10.1016/j.mri.2012.10.026.

35. Majumdar, A.e.a. Non-convex algorithm for sparse and low-rank recovery: application to dynamic MRI reconstruction. Magnetic resonance imaging 2013.

36. Lathauwer, L.D.; Moor, B.D.; Vandewalle, J. A Multilinear Singular Value Decomposition. SIAM Journal on Matrix Analysis and Applications 2000, 21, 1253-1278, doi:10.1137/s0895479896305696.

37. Zuo, X.-N.; Yu, Y.; Jin, J.; Liu, F.; Crozier, S. Multidimensional Compressed Sensing MRI Using Tensor Decomposition-Based Sparsifying Transform. PLoS ONE 2014, 9, doi:10.1371/journal.pone.0098441.

38. Beck, A.; Teboulle, M. A Fast Iterative Shrinkage-Thresholding Algorithm for Linear Inverse Problems. SIAM Journal on Imaging Sciences 2009, 2, 183-202, doi:10.1137/080716542.

39. Jiang, M.; Jin, J.; Liu, F.; Yu, Y.; Xia, L.; Wang, Y.; Crozier, S. Sparsity-constrained SENSE reconstruction: An efficient implementation using a fast composite splitting algorithm. Magnetic Resonance Imaging 2013, 31, 1218-1227, doi:10.1016/j.mri.2012.12.003. 
40. Candès, E.J.; Recht, B. Exact Matrix Completion via Convex Optimization. Foundations of Computational Mathematics 2009, 491 9, 717-772, doi:10.1007/s10208-009-9045-5.

41. Jung, H.; Ye, J.C.; Kim, E.Y. Improved k-t BLAST and k-t SENSE using FOCUSS. Phys Med Biol 2007, 52, 3201-3226, 493 doi:10.1088/0031-9155/52/11/018.

42. Jung, H.; Sung, K.; Nayak, K.S.; Kim, E.Y.; Ye, J.C. k-t FOCUSS: A general compressed sensing framework for high resolution dynamic MRI. Magnetic Resonance in Medicine 2009, 61, 103-116, doi:10.1002/mrm.21757.

43. Nam, S.; Akçakaya, M.; Basha, T.; Stehning, C.; Manning, W.J.; Tarokh, V.; Nezafat, R. Compressed sensing reconstruction for whole-heart imaging with 3D radial trajectories: a graphics processing unit implementation. Magn Reson Med 2013, 69, 91-102, doi:10.1002/mrm.24234.

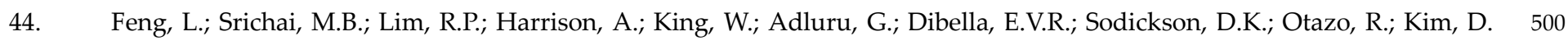
Highly accelerated real-time cardiac cine MRI usingk-tSPARSE-SENSE. Magnetic Resonance in Medicine 2013, 70, 64-74, 501 doi:10.1002/mrm.24440.

45. Paul, J.; Divkovic, E.; Wundrak, S.; Bernhardt, P.; Rottbauer, W.; Neumann, H.; Rasche, V. High-resolution respiratory 